\title{
Perfeccionismo en escolares de Caracas: diferencias EN FUNCIÓN DEL SEXO, TIPO DE INSTITUCIÓN EDUCATIVA Y NIVEL DE ESTRÉS
}

\section{Perfectionism in Schoolchildren from Caracas: Differences based on Sex, Type of Educational Institution and Stress Level}

\author{
Leonardo Andrés Aguilar Durán ${ }^{1}$
}

\section{Recibido: 2019-04-22 Aceptado: 2019-05-02}

Resumen: El objetivo de este artículo de investigación científica es caracterizar el perfeccionismo en función del sexo, el tipo de institución educativa (pública o privada) y los niveles de estrés de una muestra de escolares. Además, se analizan las diferencias en estrés entre estudiantes con alto y bajo perfeccionismo. Se trata de un estudio descriptivo-comparativo con diseño transversal. Participaron 342 escolares de Caracas $\left(M_{\text {edad }}=11.88 \pm\right.$ 0.67 ), quienes respondieron la versión venezolana de la Escala de Perfeccionismo Infantil (Oros, 2003) y el Cuestionario sobre Fuentes de Estrés Infantil (Dávila y Guarino, 2001). Se encontraron niveles moderados de perfeccionismo en la muestra. Los varones puntuaron más alto que las chicas en perfeccionismo y autodemandas. El grupo de las escuelas públicas presentó puntuaciones más altas en autodemandas específicas. En la mayoría de los casos, los participantes con alto estrés ob- tuvieron puntuaciones superiores en perfeccionismo y aquellos con alto perfeccionismo presentaron mayores puntuaciones en estrés. Estos hallazgos representan un primer acercamiento a la relación entre perfeccionismo y malestar psicológico en el contexto infantil venezolano.

Palabras clave: perfeccionismo, estrés, malestar psicológico, infancia, adolescencia

\begin{abstract}
The objective of this scientific research article is to characterize the perfectionism of a sample of school children in function of sex, type of educational institution (public or private) and stress levels. In addition, stress differences between students with high and low perfectionism are analyzed. This is a descriptive-comparative and cross-sectional study. A sample of 342 school children $\left(M_{\text {age }}=11.88 \pm 0.67\right)$ completed the Venezue-
\end{abstract}

Para citar este artículo en APA: Aguilar, L. (2019). Perfeccionismo en escolares de Caracas: Diferencias en función del sexo, tipo de institución educativa y nivel de estrés. Revista de Psicologia Universidad de Antioquia, 11(1), 7-33. DOI: https://doi. org/10.17533/udea.rp.v11n1a01
1. Licenciado en Psicología -Mención: Psicología Clínica-, Universidad Central de Venezuela (UCV), Caracas. Correo: psileonardo@gmail.com; https://orcid.org/0000-0001-95160557 
lan version of the Childhood Perfectionism Scale of Oros (2003) and the Questionnaire about Sources of Childhood Stress (Dávila y Guarino, 2001). Moderate levels of perfectionism were found in the sample. Boys scored higher than girls on perfectionism and irrational self-demands. Group of public schools presented higher scores than group of the private institutions on specific self-demands. In most cases, participants with high stress obtained higher scores on perfectionism and those with high perfectionism had higher scores on stress. These findings represent a first approach to the relationship between perfectionism and psychological distress in the Venezuelan children's context.

Keywords: Perfectionism, stress, psychological distress, childhood, adolescence

\section{Introducción}

El perfeccionismo se define como la tendencia a establecer estándares de desempeño sumamente elevados, a ejercer una autocrítica muy estricta y a medir la autovaloración en términos de logros y/o equivocaciones (Hewitt \& Flett, 1991). El estudio de este rasgo de la personalidad no ha cesado desde que en los años noventa se publicaran algunos de los instrumentos más utilizados en la actualidad para evaluarlo de forma multidimensional. Fue precisamente este avance el que permitió demostrar que, en su vertiente desadaptativa, el perfeccionismo se vincula con numerosos y graves problemas de salud mental (Hewitt et al., 2015).

Incluso cuando se sabe que el perfeccionismo puede tener un inicio temprano y a pesar de que la investigación sobre esta variable ha suscitado un gran interés desde principios de la década de los noventa, los trabajos que revisan el estado del conocimiento en el área indican que el estudio del perfeccionismo en niños y adolescentes es un campo todavía emergente (Aguilar y Castellanos, 2016; García-Fernández et al., 2016; Gonzálvez, Inglés, Lagos-San Martín, García-Fernández y Martínez-Monteagudo, 2015; Morris \& Lomax, 2014; Serppe et al., 2017). Los investigadores suelen referir como motivo principal de esta dilación, la carencia inicial de instrumentos específicamente dirigidos a la medición del perfeccionismo en estos grupos etarios (Lozano, García, Martín y Lozano, 2012; Rice \& Preusser, 2002).

En los trabajos que han tenido por objeto ofrecer un análisis bibliométrico y temático de la literatura científica internacional sobre perfeccionismo infantojuvenil (García-Fernández et al., 2016; Gonzálvez et al., 2015; Morris \& Lomax, 2014) se ha observado un claro predominio de los estudios destinados a examinar la asociación entre el perfeccionismo y diversas variables psicopa- 
tológicas, o de aquellas investigaciones que, en todo caso, centran su interés en dilucidar los aspectos negativos del constructo. En este sentido, en nińos y adolescentes se halla fuertemente asentada la relación del perfeccionismo con la depresión, la ideación suicida, la ansiedad, los trastornos de la conducta alimentaria y el trastorno obsesivo compulsivo. También se le ha relacionado con problemas que hacen parte del espectro de la salud mental como el rechazo escolar, el insomnio, los dolores de cabeza, las autolesiones, la ira y la rumia psicológica.

Queda claro entonces que parte de la importancia de investigar el perfeccionismo reside en que es un rasgo potencialmente neurótico, asociado desde las más tempranas edades con diversos problemas psicológicos y que, incluso, ha demostrado ser un factor que dificulta el abordaje terapéutico de muchas de estas perturbaciones (Halmi, 2013). Por si fuese poco, es probable que los niños con fuertes creencias perfeccionistas generen un esquema de pensamiento rígido que los acompañe a lo largo de la vida y derive en diversos trastornos psicológicos durante la adultez (Oros, 2005). De ahí la importancia de la evaluación y el tratamiento del perfeccionismo desadaptativo en las primeras etapas del ciclo vital, momentos en los cuales las relaciones paternofiliales parecen ser su caldo de cultivo (Flett, Hewitt, Oliver, \& Macdonald, 2002).

La mayoría de los modelos propuestos para la evaluación del perfeccionismo en adultos son multidimensionales, a partir de ellos se han desarrollado diversos instrumentos para evaluar el perfeccionismo en niños (Leone \& Wade, 2018). En el año 2003, Oros desarrolló en Argentina una escala para niños que evalúa el perfeccionismo disfuncional autorientado, una de las tres facetas del perfeccionismo propuestas por Hewitt \& Flett (1991). Este hito posiciona al país austral como el primero de Latinoamérica en desarrollar un instrumento de autorreporte para la evaluación del perfeccionismo en edades tempranas. La escala en cuestión permite indagar la presencia de pensamientos irracionales en forma de autodemandas y las consecuentes reacciones ante el fracaso de las mismas. Esta medida ha demostrado tener un buen funcionamiento psicométrico en población argentina y espańola, ha sido adaptada y validada para niños venezolanos (Aguilar y Castellanos, 2015) y peruanos (Ventura-León, Jara-Avalos, Garcia-Pajuelo y Ortiz-Saenz, 2018), y está siendo adaptada para 
población infantil chilena. La estructura factorial de la versión venezolana de la escala distingue entre las autodemandas específicas — puntualizan la exigencia que refieren - o absolutistas — no las puntualizan —; el malestar ante el fracaso o el hecho de "sentirse mal" al perder; $y$, los correlatos negativos de los errores, es decir, reacciones de rumia, culpa y autodescalificación ante las equivocaciones.

En contraste con la atención que ha recibido la psicopatología asociada al perfeccionismo como núcleo temático de investigación, los estudios bibliométricos (García-Fernández et al., 2016; Gonzálvez et al., 2015) han encontrado un menor número de trabajos destinados a examinar las diferencias en los niveles de perfeccionismo en función de variables demográficas, es por eso que el presente estudio comienza por considerar este tipo de aspectos.

La primera variable para tomar en cuenta es el sexo, entendido como una condición orgánica de los individuos. Las investigaciones que han analizado las posibles diferencias de perfeccionismo en función del sexo con diversas poblaciones adultas y empleando distintas medidas del constructo, han obtenido resultados dispares. Algunos trabajos encontraron diferencias significativas en los niveles de perfeccionismo a favor de los hombres (e.g., Khanijaun, 2004) o en favor de las mujeres (e.g., Arana, Keegan, y Rutsztein, 2009; Furnham $\&$ Grump, 2013). Por su parte, estudios como el de Anshel, Kim \& Henry (2009) reportaron diferencias según dimensiones evaluadas, de modo que los hombres puntuaron más alto en el factor de organización de la Escala Multidimensional de Perfeccionismo (MPS-F, por sus siglas en inglés: Frost Multidimensional Perfectionism Scale) de Frost (Marten, Lahart y Rosenblate, 1990), mientras que las expectativas y críticas paternas fueron superiores en las mujeres. En otros trabajos no se hallaron diferencias estadísticamente significativas entre ambos sexos (e.g., Amores, 2017; Anshel \& Eom, 2003; Beauregard, 2012; Blankstein, Lumley \& Crawford, 2007; Carrasco, Belloch, y Perpiñá, 2010; Çerkez, 2017; Dunn, Gotwals \& Dunn, 2005; Saboonchi \& Lundh, 2003; Thomson, 2017). Además, en varios estudios se ha conseguido una distribución similar de hombres y mujeres entre perfeccionistas adaptativos y desadaptativos (e.g., Grzegorek, Slaney, Franze \& Rice, 2004).

Esta falta de consenso también se ve reflejada en los trabajos que han empleado muestras clínicas, es decir, integradas por pacientes psiquiátricos. Así, 
mientras en algunas investigaciones los niveles de perfeccionismo no variaron significativamente por sexo (e.g., Habke, 1997), en el estudio conducido por Hewitt, Flett, Turnbull-Donovan y Mikail (1991) los hombres tuvieron niveles más altos de perfeccionismo orientado hacia los demás y las mujeres tuvieron puntajes más altos de perfeccionismo socialmente prescrito, dos dimensiones de la Escala Multidimensional de Perfeccionismo (MPS-H, por sus siglas en inglés: Hewitt Multidimensional Perfectionism Scale) de Hewitt \& Flett (1991).

La disparidad a la que se ha hecho referencia alcanza también al estudio del perfeccionismo en la infancia y la adolescencia. Un primer grupo de trabajos reúne aquellos en los que los chicos obtuvieron puntuaciones superiores a las chicas en perfeccionismo (e.g., Chemisquy, Oros, Serppe, y Ernst, 2019; Douilliez \& Hénot, 2013; Elizathe, Murawski, Custodio y Rutsztein, 2012; Oros y Vargas-Rubilar, 2016; Pamies y Quiles, 2014; Serppe, Chemisquy y Oros, 2016; Vicent et al., 2017). Por el contrario, existe un segundo grupo de estudios donde el perfeccionismo resultó significativamente superior en las chicas (e.g., Jaradat, 2013; Stornelli, Flett \& Hewitt, 2009; Uz-Baş, 2011; Yang, Hong, Tao \& Zhu, 2015). Se identifican también investigaciones que apreciaron diferencias dependiendo de las dimensiones del perfeccionismo evaluadas: por ejemplo, utilizando un instrumento basado en la MPS-F, Siegle $\&$ Schuler (2000) hallaron que los varones reportaban expectativas parentales más fuertes, mientras que las chicas expresaban más preocupación por la organización. Por último, hay estudios en los que, simplemente, los niveles de perfeccionismo no difirieron en función del sexo de niños y adolescentes (e.g., Asseraf \& Vaillancourt, 2015).

Cabe mencionar que el único estudio que ha indagado este asunto en Venezuela es el de Aguilar, Colmenares y Barroeta (2014), quienes en una muestra de estudiantes universitarios no hallaron diferencias significativas en los puntajes de perfeccionismo por sexo. No obstante, aunque hombres y mujeres no difieran en sus niveles de perfeccionismo, sí podría haber discrepancias en la forma en que este rasgo se manifiesta, en las áreas de vida que es capaz de influenciar en cada sexo, así como en su asociación con otros trastornos y variables relevantes; pero, claramente, esto es materia de una discusión que excedería el objetivo de este artículo. 
La segunda variable demográfica, esta vez de carácter contextual, a la que el presente estudio presta atención es al tipo de gestión escolar — pública o privada- que guía el funcionamiento de las instituciones donde se efectúa la enseñanza docente. Es probable que, dependiendo de la dinámica educativa que se produce en uno y otro tipo de institución, la conducta perfeccionista se vea o no alentada. Además, dado que la educación impartida en las instituciones del Estado es gratuita, sus estudiantes podrían representar una muestra particular en términos del nivel socioeconómico, variable que sin embargo no ha sido considerada en los estudios sobre perfeccionismo.

De vuelta al ámbito de lo psicopatológico, es de interés para este trabajo examinar la relación del estrés con el perfeccionismo, variables entre las cuales se ha encontrado una asociación positiva en varios estudios precedentes con estudiantes universitarios norteamericanos (e.g., Chang, Watkins, \& Banks, 2004) y chinos (Cheng, 2001), lo mismo que con niños y adolescentes de diversas nacionalidades (e.g., Butt, 2010; Muñoz, López-Mora, Portolés y González, 2016a, 2016b; O’Connor, Rasmussen \& Hawton, 2010). La literatura también ha señalado al perfeccionismo como un predictor de estrés en la adultez (Schweitzer \& Hamilton, 2002) y la adolescencia (Muñoz et al., 2016b). Otras relaciones complejas se han documentado: la predicción de la depresión y la ansiedad a partir de la interacción del perfeccionismo con el estrés (e.g., Hewitt et al., 2002), la función moderadora del perfeccionismo en la relación entre el estrés y la depresión (e.g., Cheng, 2001), y la mediación del estrés sobre el vínculo entre el perfeccionismo y el malestar psicológico (e.g., Chang et al., 2004).

En Venezuela, la relación perfeccionismo-estrés ha sido analizada en dos investigaciones. La primera fue conducida por Parra y Rodrigues (2014) en una muestra de estudiantes universitarios de Artes, determinándose que altos niveles de estrés percibido se asociaban con altos niveles de perfeccionismo académico. La segunda investigación fue la de Elvira-Valdés (2015), en la que además de comprobarse una correlación positiva entre perfeccionismo y estrés académico en estudiantes universitarios de reciente ingreso, se encontró al perfeccionismo como un predictor significativo del estrés. 
Otra de las aristas válidas para analizar la relación del perfeccionismo con el estrés es la que pasa por considerar que los perfeccionistas desadaptativos utilizan estrategias ineficaces para enfrentar las adversidades (e.g., Dunkley, Zuroff, \& Blankstein, 2003). A este respecto, la investigación de Oros (2004) demostró que, frente a situaciones amenazantes, los niños perfeccionistas hacen uso de estrategias que no consiguen disminuir el estrés, conclusión que pone de relieve el hecho de que el perfeccionismo actúa como un factor de vulnerabilidad psicológica durante la infancia y la adolescencia (Oros, 2005).

En resumen, los objetivos de este trabajo fueron los siguientes: caracterizar el perfeccionismo en una muestra de escolares caraqueños, comparar los niveles de dicho rasgo en función del sexo y del tipo de institución en la que se cursa estudios (públicas y privadas), analizar las diferencias en perfeccionismo entre estudiantes con alta y baja puntuación en estrés, y evaluar las diferencias en estrés entre los participantes con alto y bajo perfeccionismo.

\section{Método}

\section{Tipo y diseño de investigación}

Fue un estudio cuantitativo con alcance descriptivo-comparativo y diseño transversal (Hernández, Fernández, y Baptista, 2014).

\section{Participantes}

Se trabajó con una muestra intencional de 342 escolares: 163 chicos (47.7\%) y 179 chicas $(52.3 \%)$, de 11 a 15 años $(M=11.88, D T=0.67)$, cursantes del $6^{\circ}$ grado de educación primaria en ocho instituciones educativas de Caracas, cuatro públicas $(n=141,41.2 \%)$ y cuatro privadas $(n=201,58.8 \%)$.

\section{Instrumentos}

Escala de Perfeccionismo Infantil:

Se utilizó la versión adaptada y validada por Aguilar y Castellanos (2015) al contexto venezolano de la escala de Oros (2003). Consta de 15 ítems agrupa- 
dos en cuatro subescalas: Autodemandas Absolutistas, Autodemandas Específicas, Malestar ante el Fracaso y Correlatos Negativos de los Errores. El instrumento se contesta en una escala Likert de tres puntos: $1=$ no o no lo pienso, $2=$ a veces o lo pienso algunas veces, $3=$ sío lo pienso muchas veces. Un Análisis de Componentes Principales con rotación Varimax mostró que los cuatro factores explican $50.27 \%$ de la varianza total. La confiabilidad de consistencia interna evaluada mediante el coeficiente alfa resultó satisfactoria $(\alpha=.76)$.

Cuestionario sobre Fuentes de Estrés Infantil:

Se trata de la adaptación a la población venezolana (Dávila y Guarino, 2001) de la Daily Life Stressors Scale de Kearney, Drabman \& Beasley (1993). Está conformado por 62 ítems que describen posibles situaciones de tensión experimentadas por los niños en las áreas escolar, familiar, social e individual. Se responde tomando en cuenta tres niveles: $0=$ nada de malestar, $1=$ un poco de malestar y 2 = mucho malestar. La fiabilidad calculada mediante el alfa de Cronbach fue de .85 .

\section{Procedimiento}

Los estudiantes respondieron voluntariamente los instrumentos en sus aulas de clases. Previamente se tramitó la autorización de las instituciones educativas y, por tratarse de participantes menores de edad, se gestionó el consentimiento informado de sus representantes legales.

\section{Análisis de los datos}

Para la escala de perfeccionismo y sus dimensiones se calcularon medidas de tendencia central y de dispersión; a cada ítem se le calculó la media, la desviación estándar y la distribución de frecuencias. Cuando una variable necesitó dicotomizarse en bajas y altas puntuaciones, se consideraron bajas las iguales o inferiores al percentil 25 y altas aquellas iguales o superiores al percentil 75 . Los contrastes estadísticos se realizaron mediante las pruebas $t$ de Student de comparación de medias y $U$ de Mann-Whitney, según normalidad de las variables estimada a través de la prueba de Kolmogorov-Smirnov. En caso 
de diferencias estadísticamente significativas, se utilizaron — según correspondía- la $d$ de Cohen y la r de Rosenthal como medidas del tamaño del efecto. La mayoría de los cálculos se realizaron con el programa Statistical Package for the Social Sciences (SPSS), versión 20.

\section{Resultados}

\section{Análisis descriptivo de la Escala de Perfeccionismo Infantil}

En la tabla 1 se presentan los índices descriptivos de la variable de estudio y sus dimensiones. Analizados en conjunto y tomando en cuenta el rango potencial de la escala, los estadísticos indican que el grupo de participantes presentó niveles moderados de perfeccionismo. Dentro de estos valores, los más bajos correspondieron a las autodemandas específicas y los más elevados a los correlatos negativos de los errores.

Tabla 1. Estadistica descriptiva de la Escala de Perfeccionismo Infantil

\begin{tabular}{|l|c|c|c|c|c|c|c|c|}
\hline & Moda & $M d n$ & $M$ & $D E$ & As & K & Mín. & Máx. \\
\hline Perfeccionismo & 26 & 28 & 27.93 & 5.31 & 0.28 & -0.06 & 16 & 45 \\
\hline Autodemandas Absolutistas & 10 & 11 & 11.33 & 2.89 & 0.27 & -0.73 & 6 & 18 \\
\hline Autodemandas Específicas & 3 & 3 & 3.23 & 1.18 & 0.83 & -0.16 & 2 & 6 \\
\hline Malestar ante el Fracaso & 5 & 5 & 5.58 & 1.61 & 0.22 & -0.61 & 3 & 9 \\
\hline Correlatos Negativos de los Errores & 7 & 8 & 7.79 & 1.90 & 0.04 & -0.48 & 4 & 12 \\
\hline
\end{tabular}

\section{Análisis descriptivo de los items de la Escala de Perfeccionismo Infantil}

En atención a los datos de la tabla 2, puede apreciarse que las medias más altas resultaron ser las de los ítems $14(M=2.3), 10(M=2.3)$ y $2(M=2.1)$. Al considerar el porcentaje de escogencia de la alternativa 3 de la Escala de Perfeccionismo Infantil, se agrega el ítem 4 ( $\left.n_{3}=114,33.3 \%\right)$.

En cambio, los menores puntajes promedios y más bajos porcentajes de adhesión a la opción 3 correspondieron a los ítems $15\left(M=1.4, n_{3}=29,8.5\right.$ $\%), 6\left(M=1.5, n_{3}=36,10.5 \%\right)$ y $3\left(M=1.6, n_{3}=46,13.5 \%\right)$. 
Tabla 2. Descriptivos de los items de la Escala de Perfeccionismo Infantil

\begin{tabular}{|c|c|c|c|c|c|c|c|c|c|}
\hline \multirow{3}{*}{\begin{tabular}{|l} 
\\
\\
Ítems \\
\end{tabular}} & \multirow[b]{3}{*}{ Dim. } & \multirow[b]{3}{*}{ M } & \multirow[b]{3}{*}{$\mathrm{DE}$} & \multicolumn{6}{|c|}{ Opciones de respuesta } \\
\hline & & & & \multicolumn{2}{|c|}{ Opción 1} & \multicolumn{2}{|c|}{ Opción 2} & \multicolumn{2}{|c|}{ Opción 3} \\
\hline & & & & $N$ & $\%$ & $n$ & $\%$ & $n$ & $\%$ \\
\hline 1. Necesito ser el mejor & AA & 1.98 & 0.72 & 92 & 26.9 & 164 & 48.0 & 86 & 25.1 \\
\hline 2. Tengo que ser el mejor alumno & AA & 2.13 & 0.72 & 70 & 20.5 & 158 & 46.2 & 114 & 33.3 \\
\hline 3. Debo ganar siempre & AA & 1.62 & 0.71 & 175 & 51.2 & 121 & 35.4 & 46 & 13.5 \\
\hline 4. No puedo cometer errores & AA & 2.01 & 0.81 & 112 & 32.7 & 116 & 33.9 & 114 & 33.3 \\
\hline $\begin{array}{l}\text { 5. Mis trabajos deben ser mejores que los de } \\
\text { mis compañeros }\end{array}$ & AA & 1.77 & 0.74 & 140 & 40.9 & 139 & 40.6 & 63 & 18.4 \\
\hline $\begin{array}{l}\text { 6. No debo perder cuando juego con mis } \\
\text { amigos }\end{array}$ & $\mathrm{AE}$ & 1.49 & 0.68 & 210 & 61.4 & 96 & 28.1 & 36 & 10.5 \\
\hline 7. Debo ser el mejor del salón & AA & 1.82 & 0.78 & 141 & 41.2 & 123 & 36.0 & 78 & 22.8 \\
\hline 8. Debo ser el primero en terminar las tareas & $\mathrm{AE}$ & 1.74 & 0.78 & 160 & 46.8 & 112 & 32.7 & 70 & 20.5 \\
\hline 9. Cuando pierdo me siento mal & MF & 1.86 & 0.68 & 107 & 31.3 & 176 & 51.5 & 59 & 17.3 \\
\hline 10. Pienso mucho en qué me equivoqué & CNE & 2.30 & 0.75 & 59 & 17.3 & 121 & 35.4 & 162 & 47.4 \\
\hline $\begin{array}{l}\text { 11. Me siento muy mal cuando pierdo en } \\
\text { algún juego o deporte }\end{array}$ & MF & 1.72 & 0.75 & 158 & 46.2 & 123 & 36.0 & 61 & 17.8 \\
\hline $\begin{array}{l}\text { 12. Me cuesta mucho aceptar que me } \\
\text { equivoqué }\end{array}$ & $\mathrm{CNE}$ & 1.79 & 0.80 & 153 & 44.7 & 107 & 31.3 & 82 & 24.0 \\
\hline $\begin{array}{l}\text { 13. Me da rabia cuando no logro lo que } \\
\text { quiero }\end{array}$ & MF & 2.01 & 0.79 & 104 & 30.4 & 131 & 38.3 & 107 & 31.3 \\
\hline $\begin{array}{l}\text { 14. Me siento culpable cuando cometo algún } \\
\text { error }\end{array}$ & CNE & 2.32 & 0.76 & 62 & 18.1 & 109 & 31.9 & 171 & 50.0 \\
\hline 15. Me insulto cada vez que cometo un error & CNE & 1.37 & 0.64 & 243 & 71.1 & 70 & 20.5 & 29 & 8.5 \\
\hline
\end{tabular}

\section{Diferencias en perfeccionismo según el sexo y el tipo de institución educativa}

Tal como puede observarse en la tabla 3, los chicos presentaron puntuaciones significativamente más altas que las chicas en perfeccionismo $\left(t_{(340)}=2.54\right.$, $p<.05)$ y autodemandas, tanto absolutistas $(U=12289, \mathrm{Z}=-2.53, p<.05)$ como específicas $(U=12026, Z=-2.93, p<.01)$. Sin embargo, en los tres casos se reportan efectos de pequeña magnitud. Asimismo, los chicos alcanzaron un rango promedio más elevado que las chicas en cuanto a los correlatos negativos de los errores, mientras que las chicas dominaron en los niveles de malestar ante el fracaso. No obstante, estas últimas diferencias no fueron significativas desde el punto de vista estadístico $(p>.05)$. 
Perfeccionismo en escolares de Caracas: diferencias en función del sexo, tipo de institución educativa y nivel de estrés

Tabla 3. Diferencias en perfeccionismo según sexo

\begin{tabular}{|l|l|l|l|l|l|}
\hline & $\begin{array}{l}\text { Chicos } \\
(n=163)\end{array}$ & $\begin{array}{l}\text { Chicas } \\
(n=179)\end{array}$ & $t / U$ & $p$ & $d / \mathrm{r}$ \\
\hline Perfeccionismo & $28.69(5.21)$ & $27.24(5.32)$ & $t=2.54$ & .012 & $d=0.28$ \\
\hline Autodemandas Absolutistas & $12(185.61)$ & $10(158.65)$ & $U=12289.00$ & .011 & $\mathrm{r}=0.14$ \\
\hline Autodemandas Específicas & $3(187.22)$ & $3(157.18)$ & $U=12026.00$ & .003 & $\mathrm{r}=0.16$ \\
\hline Malestar ante el Fracaso & $5(168.48)$ & $6(174.25)$ & $U=14095.50$ & .583 & - \\
\hline Correlatos Negativos de los Errores & $8(180.33)$ & $8(163.46)$ & $U=13149.50$ & .111 & - \\
\hline $\begin{array}{l}\text { Nota: } \text { Datos expresados mediante media (desviación estándar) o mediana (rango promedio). } \\
\text { Pruebas de comparación: } t \text { de Student o } U \text { de Mann-Whitney. }\end{array}$ & \\
\hline
\end{tabular}

Por otro lado, como se observa en la tabla 4, al compararse los niveles de perfeccionismo según el tipo de institución donde estudiaban los participantes, se encontró una diferencia estadísticamente significativa y de efecto moderado en las autodemandas específicas $(U=9154, Z=-5.82, p<.01)$, a favor de las instituciones educativas públicas. Los estudiantes de los centros públicos también obtuvieron puntajes más elevados en perfeccionismo general y malestar ante el fracaso, mientras que los matriculados en instituciones privadas reportaron puntuaciones más altas en autodemandas absolutistas y correlatos negativos de los errores, pero ninguna de esas diferencias alcanzó significatividad estadística $(p>.05)$.

Tabla 4. Diferencias en perfeccionismo según tipo de institución educativa

\begin{tabular}{|l|l|l|l|l|l|}
\hline & \multicolumn{1}{|c|}{$\begin{array}{c}\text { Públicas } \\
(n=141)\end{array}$} & $\begin{array}{c}\text { Privadas } \\
(n=201)\end{array}$ & \multicolumn{1}{c|}{$t / U$} & $p$ & \multicolumn{1}{c|}{$d / \mathrm{r}$} \\
\hline Perfeccionismo & $28.26(5.66)$ & $27.70(5.04)$ & $t=0.95$ & .343 & - \\
\hline Autodemandas Absolutistas & $11(170.41)$ & $11(172.26)$ & $U=14017.50$ & .864 & - \\
\hline Autodemandas Específicas & $3(207.08)$ & $3(146.54)$ & $U=9154.00$ & .000 & $\mathrm{r}=0.31$ \\
\hline Malestar ante el Fracaso & $6(173.31)$ & $5(170.23)$ & $U=13915.50$ & .773 & - \\
\hline Correlatos Negativos de los Errores & $8(163.49)$ & $8(177.12)$ & $U=13041.50$ & .204 & - \\
\hline $\begin{array}{l}\text { Nota: } \text { Datos expresados mediante media (desviación estándar) o mediana (rango promedio). } \\
\text { Pruebas de comparación: } t \text { de Student o } U \text { de Mann-Whitney. }\end{array}$ \\
\hline
\end{tabular}


Diferencias en las respuestas a los items según el sexo y el tipo de institución educativa

A través de la aplicación del estadístico de Mann-Whitney se esperaba conocer si existían diferencias significativas en las respuestas aportadas a cada uno de los ítems, teniendo como variables de agrupación al sexo y al tipo de institución educativa (ver tabla 5).

Los rangos medios de los puntajes obtenidos por los chicos fueron estadísticamente superiores a los de las chicas en los ítems $3(U=11281, Z=-4.00$, $p<.01), 6(U=11696.50, \mathrm{Z}=-3.67, p<.01)$ y $11(U=13492, \mathrm{Z}=-1.30$, $p<.05)$. El tamaño del efecto fue moderado-bajo para los ítems 3 y 6 , y casi imperceptible en el ítem 11. Por su parte, las chicas solo obtuvieron rangos promedios más altos en los ítems 9 y 13, sin que por ello consiguieran significatividad.

Al hacerse la comparación por el tipo de institución, se observa que las medias de los rangos para el caso de las instituciones públicas resultaron más elevadas en ocho ítems, pero solo en tres de ellos fueron estadísticamente más altas: ítems $4(U=11287.50, \mathrm{Z}=-3.40, p<.01), 6(U=11422.50, \mathrm{Z}=-3.54$, $p<.01)$ y $8(U=9532.50, Z=-5.58, p<.01)$. El tamańo del efecto tuvo una magnitud baja en los ítems 4 y 6 , para el ítem 8 resultó moderada. En contraste, los escolares inscritos en colegios privados exhibieron puntajes más altos en siete ítems, pero únicamente en dos de ellos el estadístico arrojó significancia estadística con efectos de escasa magnitud: ítems $1(U=11187.50, Z=-3.59$, $p<.01)$ y $14(U=12181, \mathrm{Z}=-2.42, p<.05)$. 
Perfeccionismo en escolares de Caracas: diferencias en función del sexo, tipo de institución educativa y nivel de estrés

Tabla 5. Diferencias en las respuestas a los items según sexo y tipo de institución educativa

\begin{tabular}{|c|c|c|c|c|c|c|c|c|c|c|c|c|}
\hline \multirow[b]{2}{*}{ Ítems } & \multicolumn{6}{|c|}{ Diferencias según sexo } & \multicolumn{6}{|c|}{ Diferencias según tipo de institución } \\
\hline & $\begin{array}{c}\text { Chicos } \\
(n=163)\end{array}$ & $\begin{array}{c}\text { Chicas } \\
(n=179)\end{array}$ & $U$ & Z & $p$ & r & $\begin{array}{c}\text { Públicas } \\
(n=141)\end{array}$ & $\begin{array}{l}\text { Privadas } \\
(n=201)\end{array}$ & $U$ & $\mathrm{Z}$ & $p$ & r \\
\hline $1(\mathrm{AA})$ & 180.76 & 163.07 & 13079.50 & -1.79 & .074 & - & 150.34 & 186.34 & 11187.50 & -3.59 & .000 & 0.19 \\
\hline $2(\mathrm{AA})$ & 176.48 & 166.96 & 13776.50 & -0.96 & .336 & - & 161.68 & 178.39 & 12786.50 & -1.66 & .096 & - \\
\hline $3(\mathrm{AA})$ & 191.79 & 153.02 & 11281.00 & -4.00 & .000 & 0.22 & 173.45 & 170.13 & 13895.00 & -0.34 & .735 & - \\
\hline $4(\mathrm{AA})$ & 176.44 & 167.00 & 13782.50 & -0.94 & .349 & - & 191.95 & 157.16 & 11287.50 & -3.40 & .001 & 0.18 \\
\hline $5(\mathrm{AA})$ & 178.48 & 165.15 & 13451.00 & -1.35 & .179 & - & 176.70 & 167.85 & 13437.50 & -0.88 & .379 & - \\
\hline $6(\mathrm{AE})$ & 189.24 & 155.34 & 11696.50 & -3.67 & .000 & 0.20 & 190.99 & 157.83 & 11422.50 & -3.54 & .000 & 0.19 \\
\hline 7 (AA) & 176.68 & 166.78 & 13744.00 & -0.99 & .322 & - & 167.59 & 174.25 & 13618.50 & -0.66 & .511 & - \\
\hline $8(\mathrm{AE})$ & 177.08 & 166.42 & 13678.50 & -1.08 & .281 & - & 204.39 & 148.43 & 9532.50 & -5.58 & .000 & 0.30 \\
\hline $9(\mathrm{MF})$ & 165.63 & 176.85 & 13631.50 & -1.15 & .249 & - & 166.65 & 174.90 & 13486.50 & -0.84 & .404 & - \\
\hline $10(\mathrm{CNE})$ & 180.30 & 163.49 & 13154.00 & -1.71 & .087 & - & 161.06 & 178.82 & 12699.00 & -1.78 & .075 & - \\
\hline $11(\mathrm{MF})$ & 178.23 & 165.37 & 13492.00 & -1.30 & .019 & 0.07 & 178.83 & 166.36 & 13137.50 & -1.25 & .213 & - \\
\hline $12(\mathrm{CNE})$ & 179.87 & 163.88 & 13224.00 & -1.61 & .108 & - & 169.52 & 172.89 & 13891.50 & -0.33 & .739 & - \\
\hline 13 (MF) & 163.94 & 178.38 & 13357.00 & -1.43 & .152 & - & 172.99 & 170.46 & 13960.50 & -0.25 & .804 & - \\
\hline $14(\mathrm{CNE})$ & 174.46 & 168.80 & 14106.00 & -0.58 & .564 & - & 157.39 & 181.40 & 12181.00 & -2.42 & .016 & 0.13 \\
\hline 15 (CNE) & 172.99 & 170.14 & 14345.00 & -0.34 & .737 & - & 178.32 & 166.71 & 13208.50 & -1.34 & .179 & - \\
\hline
\end{tabular}

\section{Diferencias en perfeccionismo en función de los niveles de estrés}

Se evaluaron las diferencias en perfeccionismo entre los escolares con mayor y menor estrés. Para ello, se comparó a los participantes cuya puntuación total en el Cuestionario sobre Fuentes de Estrés Infantil se situaba en el primer cuartil con aquellos cuya puntuación en este instrumento estaba en el tercer cuartil. En la tabla 6 es posible observar que, en dieciocho de los veinticinco conjuntos de comparación calculados, los estudiantes con altos niveles de estrés presentaron puntuaciones significativamente más altas en perfeccionismo que sus iguales con bajo estrés $(p<.05, p<.01)$. Los efectos fueron entre moderados y grandes. Solamente en un caso (autodemandas específicas en escolares de instituciones privadas) la puntuación en perfeccionismo resultó superior en los participantes con bajo estrés, pero esa diferencia no fue significativa $(p>.05)$. 
Tabla 6. Diferencias en perfeccionismo según niveles de estrés

\begin{tabular}{|c|c|c|c|c|c|}
\hline & Estrés $\left(\mathrm{P}_{25}\right)$ & Estrés $\left(\mathrm{P}_{75}\right)$ & $t / U$ & $p$ & $d / \mathrm{r}$ \\
\hline Perfeccionismo & $25.42(5.66)$ & $30.69(5.20)$ & $t=-4.55$ & .000 & $d=0.97$ \\
\hline Chicos & $26.55(4.77)$ & $32.78(6.30)$ & $t=-2.94$ & .007 & $d=1.11$ \\
\hline Chicas & $24.43(6.27)$ & $30.17(4.85)$ & $t=-3.95$ & .000 & $d=1.02$ \\
\hline Instituciones públicas & $25.13(5.76)$ & $31.22(5.17)$ & $t=-3.99$ & .000 & $d=1.11$ \\
\hline Instituciones privadas & $25.79(5.67)$ & $29.89(5.29)$ & $t=-2.27$ & .029 & $d=0.74$ \\
\hline Autodemandas Absolutistas & $10(36.01)$ & $13(52.61)$ & $U=602.50$ & .002 & $r=0.33$ \\
\hline Chicos & $11.20(2.63)$ & $13.00(2.40)$ & $t=-1.75$ & .091 & - \\
\hline Chicas & $9(22.09)$ & $13(35.06)$ & $U=232.00$ & .004 & $r=0.37$ \\
\hline Instituciones públicas & $10.46(3.16)$ & $12.15(2.98)$ & $t=-1.96$ & .055 & - \\
\hline Instituciones privadas & $10(14.82)$ & $13(23.42)$ & $U=91.50$ & .015 & $r=0.40$ \\
\hline Autodemandas Específicas & $3(39.07)$ & $3(49.69)$ & $U=734.00$ & .044 & $r=0.22$ \\
\hline Chicos & $3(13.85)$ & $5(17.56)$ & $U=67.00$ & .265 & - \\
\hline Chicas & $3(24.59)$ & $3(33.46)$ & $U=289.50$ & .045 & $r=0.26$ \\
\hline Instituciones públicas & $3(20.29)$ & $4(31.07)$ & $U=187.00$ & .008 & $r=0.37$ \\
\hline Instituciones privadas & $3(19.05)$ & $3(18.94)$ & $U=170.00$ & .974 & - \\
\hline Malestar ante el Fracaso & $5(33.15)$ & $6(55.34)$ & $U=479.50$ & .000 & $r=0.44$ \\
\hline Chicos & $4(11.90)$ & $8(21.89)$ & $U=28.00$ & .003 & $r=0.56$ \\
\hline Chicas & $5(23.63)$ & $6(34.07)$ & $U=267.50$ & .020 & $r=0.30$ \\
\hline Instituciones públicas & $4(18.04)$ & $6(33.07)$ & $U=133.00$ & .000 & $r=0.51$ \\
\hline Instituciones privadas & $5(15.76)$ & $5.50(22.42)$ & $U=109.50$ & .054 & - \\
\hline Correlatos Negativos de los Errores & $7(37.27)$ & $8(51.41)$ & $U=656.50$ & .008 & $r=0.28$ \\
\hline Chicos & $7.50(13.53)$ & $9(18.28)$ & $U=60.50$ & .159 & - \\
\hline Chicas & $7(22.54)$ & $8(34.76)$ & $U=242.50$ & .007 & $r=0.35$ \\
\hline Instituciones públicas & $6.79(1.74)$ & $8.22(2.01)$ & $t=-2.70$ & .009 & $d=0.76$ \\
\hline Instituciones privadas & $7(16.87)$ & $7.50(21.25)$ & $U=130.50$ & .212 & - \\
\hline \multicolumn{6}{|c|}{$\begin{array}{l}\text { Nota: } \mathrm{P}_{25}=25 \% \text { inferior de las frecuencias (grupo con bajo estrés). } \mathrm{P}_{75}=25 \% \text { superior de las frecuencias (grupo con } \\
\text { alto estrés). Datos expresados mediante media (desviación estándar) o mediana (rango promedio). Pruebas de compara- } \\
\text { ción: } t \text { de Student o } U \text { de Mann-Whitney. }\end{array}$} \\
\hline
\end{tabular}

\section{Diferencias en estrés en función de los niveles de perfeccionismo}

Siguiendo un procedimiento similar al anterior, se analizó si los escolares con altos niveles de perfeccionismo (puntajes $\geq$ al centil 75) diferían de sus pares con bajos niveles de perfeccionismo (puntajes $\leq$ al centil 25) en las puntuaciones en estrés. Como puede verse en la tabla 7 , los resultados de estos análisis mostraron que, en quince series de comparación, el grupo de participantes que reportó niveles elevados de perfeccionismo puntuó significativamente más alto en estrés que el grupo con bajo perfeccionismo $(p<.05, p<.01)$. Los efectos 
Perfeccionismo en escolares de Caracas: diferencias en función del sexo, tipo de institución educativa y nivel de estrés

presentaron magnitudes de moderadas a altas. El estrés familiar fue la única dimensión para la que en ningún caso existieron diferencias significativas entre escolares con alto y bajo perfeccionismo. Solo en un par de veces (estrés social en escolares de instituciones privadas y estrés familiar en chicos) las puntuaciones en estrés fueron más altas en los participantes con menor perfeccionismo, pero esas diferencias no resultaron estadísticamente significativas $(p>.05)$.

Tabla 7. Diferencias en estrés según niveles de perfeccionismo

\begin{tabular}{|c|c|c|c|c|c|}
\hline & $\begin{array}{c}\text { Perfeccionismo } \\
\left(\mathrm{P}_{25}\right)\end{array}$ & $\begin{array}{c}\text { Perfeccionismo } \\
\left(\mathrm{P}_{75}\right)\end{array}$ & $t / U$ & $p$ & $d / \mathrm{r}$ \\
\hline Estrés & $46(34.11)$ & $58.50(54.30)$ & $U=537.50$ & .000 & $r=0.39$ \\
\hline Chicos & $41(14.54)$ & $56(23.37)$ & $U=98.00$ & .025 & $r=0.35$ \\
\hline Chicas & $46.50(19.30)$ & $66(32.60)$ & $U=134.50$ & .001 & $r=0.46$ \\
\hline Instituciones públicas & $42(15.71)$ & $60(28.07)$ & $U=114.00$ & .003 & $r=0.44$ \\
\hline Instituciones privadas & $47.33(15.91)$ & $56.63(12.76)$ & $t=-2.07$ & .045 & - \\
\hline Estrés Escolar & $9(33.04)$ & $16.50(55.22)$ & $U=493.50$ & .000 & $r=0.43$ \\
\hline Chicos & $8.31(5.59)$ & $15.11(6.53)$ & $t=-3.23$ & .003 & $d=1.05$ \\
\hline Chicas & $10.96(5.52)$ & $16.67(5.80)$ & $t=-3.50$ & .001 & $d=1.01$ \\
\hline Instituciones públicas & $8.88(5.74)$ & $16.07(5.79)$ & $t=-4.08$ & .000 & $d=1.23$ \\
\hline Instituciones privadas & $11.00(5.47)$ & $15.37(6.94)$ & $t=-2.31$ & .026 & $d=0.70$ \\
\hline Estrés Familiar & $13(41.52)$ & $15(47.97)$ & $U=841.50$ & .240 & - \\
\hline Chicos & $13(20.65)$ & $14(20.43)$ & $U=173.50$ & .954 & - \\
\hline Chicas & $15(21.55)$ & $17(29.60)$ & $U=197.50$ & .050 & - \\
\hline Instituciones públicas & $10(19.47)$ & $15(25.86)$ & $U=178.00$ & .118 & - \\
\hline Instituciones privadas & $16(21.69)$ & $15(22.39)$ & $U=220.50$ & .854 & - \\
\hline Estrés Social & $8(39.85)$ & $8.50(49.40)$ & $U=773.00$ & .081 & - \\
\hline Chicos & $6.38(4.65)$ & $7.89(3.76)$ & $t=-1.10$ & .279 & - \\
\hline Chicas & $7.57(3.85)$ & $10.05(3.14)$ & $t=-2.41$ & .020 & $d=0.70$ \\
\hline Instituciones públicas & $6.53(4.43)$ & $9.66(3.60)$ & $t=-2.61$ & .012 & $d=0.78$ \\
\hline Instituciones privadas & $8(22.40)$ & $7(21.50)$ & $U=218.50$ & .815 & - \\
\hline Estrés Individual & $15(35.06)$ & $20.50(53.49)$ & $U=576.50$ & .001 & $r=0.36$ \\
\hline Chicos & $14(14.77)$ & $20(23.26)$ & $U=101.00$ & .031 & $r=0.34$ \\
\hline Chicas & $15.79(5.55)$ & $20.10(5.82)$ & $t=-2.63$ & .011 & $d=0.76$ \\
\hline Instituciones públicas & $15.47(7.43)$ & $19.41(5.70)$ & $t=-2.02$ & .049 & - \\
\hline Instituciones privadas & $14.63(5.53)$ & $19.05(5.37)$ & $t=-2.64$ & .012 & $d=0.81$ \\
\hline \multicolumn{6}{|c|}{$\begin{array}{l}\text { Nota: } \mathrm{P}_{25}=25 \% \text { inferior de las frecuencias (grupo con bajo perfeccionismo). } \mathrm{P}_{75}=25 \% \text { superior de las frecuencias } \\
\text { (grupo con alto perfeccionismo). Datos expresados mediante media (desviación estándar) o mediana (rango promedio). } \\
\text { Pruebas de comparación: } t \text { de Student o } U \text { de Mann-Whitney. }\end{array}$} \\
\hline
\end{tabular}




\section{Discusión}

La finalidad de este estudio fue describir el perfeccionismo de los escolares caraqueños atendiendo a dos variables demográficas (sexo y tipo de institución educativa) y una relativa al malestar psicológico (estrés), así como examinar las diferencias en estrés entre los alumnos con alto y bajo perfeccionismo.

Además de evidenciar la relevancia del constructo medido en la población infantojuvenil, se puede afirmar que los valores moderados de perfeccionismo obtenidos por los participantes (considerando el rango posible de la escala) representan cierto riesgo al bienestar, debido a los desórdenes emocionales con los que aquél se vincula, los cuales pueden poner en peligro el buen desarrollo psicosocial de los escolares.

El análisis de ítems indicó que las principales manifestaciones de perfeccionismo en los niños son las metas autoimpuestas de ser el mejor alumno y de no equivocarse o cometer errores, ambas aspiraciones de carácter irracional, dogmático y absoluto; mientras que la culpa y la rumiación destacaron entre las reacciones negativas que con mayor frecuencia experimentan los niños ante el incumplimiento o fracaso de sus objetivos. Vale destacar que estos resultados coinciden con los obtenidos en el medio argentino (Chemisquy et al., 2019).

El análisis detallado de los ítems también conduce a pensar que una fuerte autocrítica manifestada en forma de insultos o reproches a sí mismo, al igual que la exigencia de no perder (sobre todo si el contexto es lúdico y de camaradería), expresa un perfeccionismo extremo. Esto se afirma debido a que los ítems contentivos de tales actitudes (No 3, 6 y 15) obtuvieron las medias más bajas. Es de resaltar que en el caso argentino (Chemisquy et al., 2019) el análisis de medias también ubicó los ítems 3 y 6 entre los reactivos cuya opción más extrema fue elegida por un bajo número de niños.

En cuanto a las comparaciones por sexo, los chicos puntuaron más alto que las chicas en perfeccionismo y en los dos tipos de autodemandas, absolutistas y específicas. Estos resultados son consistentes con los reportados en otras investigaciones con adultos universitarios (Hewitt \& Flett, 1991) y población infantojuvenil francesa (Douilliez \& Hénot, 2013), española (Pamies y Quiles, 2014; Vicent et al., 2017) y argentina (Chemisquy et al., 2019; Elizathe 
et al., 2012; Oros y Vargas-Rubilar, 2016; Serppe et al., 2016), en las cuales se determinó que los niveles de perfeccionismo autorientado suelen ser más elevados en el sexo masculino.

Siguiendo lo planteado por Oros y Vargas-Rubilar (2016), se está de acuerdo en interpretar las diferencias expuestas a la luz de los condicionantes socioculturales que rodean a hombres y mujeres, los cuales se expresan en el tipo de exigencias que predomina en cada género. En tal sentido, el análisis de ítems develó que las diferencias más marcadas entre chicos y chicas se produjeron en ítems cuyo contenido gira en torno al ámbito lúdico-deportivo, donde es conocida la tendencia a la competitividad que el género masculino presenta. Así, aunque Dunn et al. (2005) no encontraron diferencias en los niveles de perfeccionismo general de hombres y mujeres, informaron que los hombres puntuaron más alto en el perfeccionismo específicamente enfocado en el deporte. Se debe tener presente que, durante la edad escolar y estando a las puertas de la adolescencia, la posesión de características típicamente asociadas con el rol masculino (e.g., fuerza, velocidad) aumentan la probabilidad que tienen los chicos de obtener aceptación social; por el contrario, los que se distancian del perfil que la sociedad presenta como exitoso, son más propensos a recibir burlas de sus compañeros (Adams \& Govender, 2008; Fletcher, Neumeister, $\&$ Flett, 2014). Ante este panorama, resulta obvio que podría incrementarse el deseo de los nińos por alcanzar la perfección que el contexto social demanda.

En referencia al tipo de institución educativa, fueron los alumnos de las escuelas públicas los que obtuvieron puntuaciones superiores en la subescala de autodemandas específicas y en tres ítems del grupo de autodemandas. Si bien es cierto que quienes asisten a instituciones de gestión privada obtuvieron mayores puntuaciones en dos ítems, los mismos pertenecen a dimensiones distintas del perfeccionismo y no es sencillo establecer una conclusión al respecto, así que por el momento solo queda mantener este resultado como un referente para indagaciones posteriores. El único estudio que ha analizado el perfeccionismo infantil a la luz del tipo de escuela es el de Chemisquy et al. (2019) en Argentina: coincidiendo parcialmente con los hallazgos aquí reportados, el resultado de la investigación arrojó que los niños de escuelas estatales tenían mayores puntuaciones en perfeccionismo que sus compañeros de las 
escuelas privadas, produciéndose el impacto de la diferencia únicamente en la dimensión de autodemandas.

En principio, se tendería a pensar que la educación primaria que siguen los niños de las instituciones privadas es más exigente. Sin embargo, es preciso colocar la lupa en las características de esa exigencia. Así, es probable que la escuela pública favorezca exigencias más disfuncionales, esto es, con un carácter más dogmático y exagerado, como las incluidas por la Escala de Perfeccionismo de Oros (2003). La razón por la cual la escuela privada favorecería exigencias más razonables no está clara, Chemisquy et al. (2019) aducen que los docentes de las instituciones privadas están mejor capacitados, pero esta no parece ser la mejor explicación en el contexto venezolano actual donde, salvo excepciones referidas a rigurosos procesos de selección que llevan a cabo reconocidos colegios capitalinos, el personal docente de las distintas escuelas es prácticamente 'el mismo'. Más bien, se piensa que las diferentes políticas institucionales son capaces de vehiculizar una actuación docente con un determinado estilo de exigencias para con los estudiantes.

Por otra parte, considerar las diferencias socioeconómicas que suelen existir entre los alumnos de la educación pública y privada puede ser una vía para explicar las diferencias encontradas en el perfeccionismo. Aunque no se han hallado estudios que relacionen directamente esta variable con el nivel socioeconómico, se sabe que las condiciones socioeconómicas adversas impactan de forma negativa en la salud mental de las personas. En este sentido, Rodríguez-Jiménez, Blasco y Piqueras (2014) encontraron que entre los adolescentes españoles con bajo nivel socioeconómico era mayor la sintomatología obsesivo-compulsiva, un cuadro cuya relación con el perfeccionismo se halla fuertemente asentada en la literatura, al punto de constituir este un criterio diagnóstico para el trastorno de la personalidad obsesivo-compulsiva según la quinta edición del Manual diagnóstico y estadístico de los trastornos mentales o DSM-5 por sus siglas en inglés [Diagnostic and Statistical Manual of Mental Disorders] (Asociación Americana de Psiquiatría, 2014). También es posible que en los estratos sociales más deprimidos de la población prevalezcan estilos parentales de crianza corresponsables de la formación de autodemandas disfuncionales durante la infancia. De todos modos, es tarea pendiente 
comprobar si realmente las variables socioeconómicas afectan los niveles de perfeccionismo, aspecto que en esta investigación no se determinó dada la obsolescencia de los métodos tradicionales para la identificación del estrato social de la familia venezolana en la actualidad y también debido a la dificultad que suponía para los participantes en edad escolar el hecho de referir la información solicitada (e.g., ingreso familiar).

Seguidamente, los esfuerzos se centraron en analizar la relación entre el perfeccionismo y una variable que ha probado perjudicar la adaptación psicológica, el estrés. Tal como se esperaba, los escolares con mayor estrés presentaron mayores puntuaciones en perfeccionismo que aquellos con menor estrés. Asimismo, en la mayoría de los casos se comprobó que quienes puntuaban alto en perfeccionismo tenían mayores puntuaciones en estrés que los escolares con bajo perfeccionismo.

Estos hallazgos están en consonancia con los de otros estudios con niños y adolescentes españoles (Muñoz et al., 2016a, 2016b), canadienses (e.g., Hewitt et al., 2002), pakistaníes (Butt, 2010) y escoceses (O’Connor et al., 2010). En ellos, desde los modelos de perfeccionismo que ofrecen la MPS-F y la Escala de Perfeccionismo para Niños y Adolescentes (CAPS, por sus siglas en inglés: Child-Adolescent Perfectionism Scale) de Flett, Hewitt, Boucher, Davidson y Munro (2000), se determinó que los indicadores de perfeccionismo desadaptativo están vinculados con el estrés, lo que señala a estas tendencias perfeccionistas como una problemática que debe ser objeto de atención clínica temprana para prevenir la aparición de trastornos asociados. De entre los antecedentes destacados, vale la pena traer a colación la investigación de Butt (2010). Esta autora, además de comprobar la asociación entre estrés y perfeccionismo mediante correlaciones bivariadas, empleó una metodología similar a la que aquí se siguió y probó que existían diferencias en estrés entre los grupos de perfeccionismo alto y bajo, de modo tal que los jóvenes que tenían puntuaciones altas en perfeccionismo también las tenían en estrés.

Ahora bien, como se seńaló oportunamente, en este estudio los grupos de perfeccionismo bajo y alto no difirieron en sus niveles de estrés familiar. Este resultado se sale de lo esperado porque la mayoría de los estudios sitúan la génesis del perfeccionismo en las experiencias tempranas del sujeto en su 
entorno familiar (e.g., Flett et al., 2002). No obstante, una revisión del contenido de los ítems del Cuestionario de Fuentes de Estrés Infantil conduce a apreciar que el dominio familiar incluye estresores relacionados con amenazas a la integridad de algún ser querido (e.g., ítem 6), conflictos del grupo familiar (e.g., ítem 41) y la realización de tareas indeseadas (e.g., ítem 47), situaciones que se alejan de la presión y las críticas que nińos y adolescentes pueden recibir por parte de sus padres y demás familiares.

Entre las limitaciones que este trabajo presenta, cabe destacar el uso exclusivo de instrumentos de autoinforme para la recolección de los datos sobre las variables psicológicas. Tales medidas, además de tener aspectos mejorables desde el punto de vista psicométrico, pueden ser sensibles a sesgos en la información aportada por los participantes y limitar la comprensión de la dinámica que se produce entre los constructos evaluados. Otras limitantes son el tamaño de la muestra y no haber seguido un muestreo probabilístico en su selección. También puede tenerse como limitación el hecho de haber empleado un diseño descriptivo-comparativo que no permite establecer relaciones de causalidad entre las variables, y cuyos resultados se deben interpretar con cautela en aquellos casos donde los tamaños del efecto observados fueron pequeños.

Además de atender los señalamientos expuestos, sería interesante que en una futura investigación se llevara a cabo un análisis diferenciado del perfeccionismo por ciclo o nivel educativo. Es asimismo tarea pendiente la elaboración de un baremo local con una muestra lo suficientemente amplia y representativa como para proveer valores de referencia que permitan interpretar los puntajes de perfeccionismo en el caso venezolano, pues en el estudio que este artículo reporta solo ha sido posible realizar una interpretación descriptiva de dichos puntajes atendiendo al rango teórico de la escala empleada. Finalmente, se destaca la importancia de continuar develando la naturaleza del perfeccionismo en los niños y adolescentes venezolanos. 
Perfeccionismo en escolares de Caracas: diferencias en función del sexo, tipo de institución educativa y nivel de estrés

\section{Referencias}

Adams, L., \& Govender, K. (2008). “Making a Perfect Man”: Traditional Masculine Ideology and Perfectionism among Adolescent Boys. South African Journal of Psychology, 38(3), 551-562. doi: 10.1177/008124630803800309

Aguilar, L., y Castellanos, M. (2015). Adaptación y validación de una escala de perfeccionismo infantil en niños venezolanos. (Trabajo de grado inédito). Universidad Central de Venezuela, Caracas.

Aguilar, L., y Castellanos, M. (2016). Perfeccionismo infantil: una revisión de la literatura. Ajayu, 14(2), 162-226. Recuperado de http://www.ucb.edu.bo/publicaciones/ajayu/v14n2/v14n2a01.html

Aguilar, L., Colmenares, D., y Barroeta, G. (2014). Consideraciones respecto a las normas de una medida de perfeccionismo académico para estudiantes universitarios. Revista de Estudios Clínicos e Investigación Psicológica, 4(7), 4-13. Recuperado de http://www.revistacecip.com/index.php/recip/article/view/54

Amores, A. (2017). Perfeccionismo, miedo al fracaso y sintomas depresivos. (Tesis de maestría). Universidad Pontificia Comillas, Madrid, España. Recuperada de: http://repositorio.comillas.edu/xmlui/handle/11531/23179

Anshel, M., \& Eom, H. (2003). Exploring the dimensions of perfectionism in sport. International Journal of Sport Psychology, 34(3), 255-271.

Anshel, M., Kim, J., \& Henry, R. (2009). Reconceptualizing indicants of sport perfectionism as a function of gender. Journal of Sport Behavior, 32(4), 395-418.

Arana, F., Keegan, E., y Rutsztein, G. (2009). Adaptación de una medida multidimensional de perfeccionismo: la Almost Perfect Scale-Revised (APS-R). Un estudio preliminar sobre sus propiedades psicométricas en una muestra de estudiantes universitarios argentinos. Evaluar, 9, 35-53. Recuperado de http:// revistas.unc.edu.ar/index.php/revaluar/article/view/463

Asociación Americana de Psiquiatría (2014). Manual diagnóstico y estadístico de los trastornos mentales, DSM-5. (5 ed.). Madrid: Editorial Médica Panamericana.

Asseraf, M., \& Vaillancourt, T. (2015). Longitudinal link between perfectionism and depression in children. Journal of Abnormal Child Psychology, 43(5), 895-908. doi: $10.1007 /$ s10802-014-9947-9

Beauregard, A. (2012). Perfectionism, self-efficacy and OCB: the moderating role of gender. Personnel Review, 41(5), 590-608. doi: 10.1108/00483481211249120 
Blankstein, K., Lumley, C., \& Crawford, A. (2007). Perfectionism, Hopelessness, And Suicide Ideation: Revisions to Diathesis-Stress and Specific Vulnerability Models. Journal of Rational-Emotive and Cognitive-Behavior Therapy, 25(4), 279-319. doi: 10.1007/s10942-007-0053-6

Butt, F. (2010). The role of perfectionism in psychological health: A study of adolescents in Pakistan. Europe's Journal of Psychology, 6(4), 125-147. doi: 10.5964/ ejop.v6i4.227

Carrasco, A., Belloch, A., y Perpiñá, C. (2010). La evaluación del perfeccionismo: utilidad de la Escala Multidimensional de Perfeccionismo en población española. Análisis y Modificación de Conducta, 36(153), 49-65. Recuperado de http:// www.uhu.es/publicaciones/ojs/index.php/amc/article/view/1225.

Çerkez, Y. (2017). The Effect of Attachment Styles on Perfectionism in Romantic Relationships. EURASIA Journal of Mathematics, Science and Technology Education, 13(10), 6923-6931. doi: 10.12973/ejmste/78539

Chang, E., Watkins, A., \& Banks, K. (2004). How Adaptive and Maladaptive Perfectionism Relate to Positive and Negative Psychological Functioning: Testing a Stress-Mediation Model in Black and White Female College Students. Journal of Counseling Psychology, 51(1), 93-102. doi: 10.1037/0022-0167.51.1.93

Chemisquy, S., Oros, L., Serppe, M., y Ernst, C. (2019). Caracterización del perfeccionismo disfuncional en la niñez tardía. Apuntes Universitarios, 9(2), 1-26. doi: 10.17162/au.v9i2.355

Cheng, S. (2001). Life Stress, Problem Solving, Perfectionism, and Depressive Symptoms in Chinese. Cognitive Therapy and Research, 25(3), 303-310. doi: 10.1023/a:1010788513083

Dávila, B., y Guarino, L. (2001). Fuentes de estrés y estrategias de afrontamiento en escolares venezolanos. Interamerican Journal of Psychology, 35(1), 97-112. Recuperado de http://www.redalyc.org/articulo.oa?id=28435105

Douilliez, C., \& Hénot, E. (2013). Mesures du perfectionnisme chez l'adolescent: validation des versions francophones de deux questionnaires. Revue Canadienne des Sciences du Comportement, 45(1), 64-71. doi: 10.1037/a0022686

Dunkley, D., Zuroff, D., \& Blankstein, K. (2003). Self-critical perfectionism and daily affect: dispositional and situational influences on stress and coping. Journal of Personality and Social Psychology, 84(1), 234-252. doi: 10.1037/00223514.84.1.234 

institución educativa y nivel de estrés

Dunn, J., Gotwals, J., \& Dunn, J. (2005). An examination of the domain specificity of perfectionism among intercollegiate student-athletes. Personality and Individual Differences, 38(6), 1439-1448. doi: 10.1016/j.paid.2004.09.009

Elizathe, L., Murawski, B., Custodio, J., y Rutsztein, G. (2012). Riesgo de trastorno alimentario en niños escolarizados de Buenos Aires: su asociación con perfeccionismo. Revista Mexicana de Trastornos Alimentarios, 3, 106-120. Recuperado de http://journals.iztacala.unam.mx/index.php/amta/article/view/226

Elvira-Valdés, M. (2015). Perfil familiar y psicológico como predictores del estrés académico: un modelo de ruta. (Tesis doctoral inédita). Universidad Simón Bolívar, Venezuela.

Fletcher, K., Neumeister, K., \& Flett, G. (2014). Introduction to the Special Issue: Perfectionism in the School Context. Psychology in the School, 51(9), 897-898. doi: $10.1002 /$ pits. 21790

Flett, G., Hewitt, P., Boucher, D., Davidson, L., \& Munro, I. (2000). The Child-Adolescent Perfectionism Scale: Development, validation, and association with adjustment. Manuscrito inédito, York University, Toronto, Canadá.

Flett, G., Hewitt, P., Oliver, J., \& Macdonald, S. (2002). Perfectionism in children and their parents: a developmental analysis. En G. Flett \& P. Hewitt (Eds.), Perfectionism: Theory, research and treatment (pp. 89-132). Washington, DC: American Psychological Association.

Frost, R., Marten, P., Lahart, C., \& Rosenblate, R. (1990). The dimensions of perfectionism. Cognitive Therapy and Research, 14(5), 449-468. doi: 10.1007/ BF01172967

Furnham, A., \& Grump, J. (2013). The sensitive, imaginative, articulate art student and conservative, cool, numerate science student: individual differences in art and science students. Learning and Individual Differences, 25, 150-155. doi: 10.1016/j.lindif.2013.03.002

García-Fernández, J., Inglés, C., Vicent, M., Gonzálvez, C., Gómez-Núñez, M., y Poveda-Serra, P. (2016). Perfeccionismo durante la infancia y la adolescencia. Análisis bibliométrico y temático (2004-2014). Revista Iberoamericana de Psicología y Salud, 7(2), 79-88. doi: 10.1016/j.rips.2016.02.001

Gonzálvez, C., Inglés, C., Lagos-San Martín, N., García-Fernández, J., y Martínez-Monteagudo, M. (2015). Análisis bibliométrico sobre el perfeccionismo en la infancia y la adolescencia. International Journal of Developmental and Educational Psychology, 1(1), 211-218. doi: 10.17060/ijodaep.2015.n1.v1.48 
Grzegorek, J., Slaney, R., Franze, S., \& Rice, K. (2004). Self-Criticism, Dependency, Self-Esteem, and Grade-point Average Satisfaction among Clusters of Perfectionists and Nonperfectionists. Journal of Counseling Psychology, 51(2), 192-200. doi: 10.1037/0022-0167.51.2.192

Habke, A. (1997). The manifestations of perfectionistic self-presentation in a clinical sample. (Tesis doctoral). University of British Columbia, Vancouver, Canadá. Recuperada de http://open.library.ubc.ca/cIRcle/collections/ubctheses/831/ items/1.0099204

Halmi, K. (2013). Perplexities of treatment resistance in eating disorders. BMC Psychiatry, 13(292). doi: 10.1186/1471-244X-13-292

Hernández, R., Fernández, C., y Baptista, P. (2014). Metodología de la investigación. (6 ${ }^{\mathrm{a}}$ ed.). México: McGraw-Hill Interamericana.

Hewitt, P., \& Flett, G. (1991). Perfectionism in the self and social contexts: Conceptualization, assessment, and association with psychopathology. Journal of Personality and Social Psychology, 60(3), 456-470. doi: 10.1037/0022-3514.60.3.456

Hewitt, P., Caelian, C., Flett, G., Sherry, S., Collins, L., \& Flynn, C. (2002). Perfectionism in children: Association with depression, anxiety, and anger. Personality and Individual Differences, 32(6), 1049-1061. doi: 10.1016/s01918869(01)00109-x

Hewitt, P., Flett, G., Turnbull-Donovan, W., \& Mikail, S. (1991). The Multidimensional Perfectionism Scale: Reliability, validity, and psychometric properties in psychiatric samples. Psychological Assessment, 3(3), 464-468. doi: 10.1037/10403590.3.3.464

Hewitt, P., Mikail, S., Flett, G., Tasca, G., Flynn, C., Deng, X., Chen, C. (2015). Psychodynamic/interpersonal group psychotherapy for perfectionism: Evaluating the effectiveness of a short-term treatment. Psychotherapy, 52(2), 205-217. doi: $10.1037 /$ pst0000016

Jaradat, A. (2013). Multidimensional perfectionism in a sample of Jordanian high school students. Australian Journal of Guidance and Counselling, 23(1), 95-105. doi: $10.1017 /$ jgc.2012.20

Kearney, C., Drabman, R., Beasley, J. (1993). The trials of childhood: The development, reliability, and validity of the Daily Life Stressors Scale. Journal of Child and Family Studies, 2(4), 371-388. doi: 10.1007/BF01321232

Khanijaun, S. (2004). Gender differences in perfectionism and hardiness in relation to suicide ideation among Assumption University BBA undergraduates. (Tesis de 
maestría). Assumption University, Tailandia). Recuperada de http://repository. au.edu/handle/6623004553/1228

Leone, E., \& Wade, T. (2018). Measuring perfectionism in children: a systematic review of the mental health literature. European Child and Adolescent Psychiatry, 27(5), 553-567. doi: 10.1007/s00787-017-1078-8

Lozano, L., García, E., Martín, M., y Lozano, L. (2012). Desarrollo y validación del Inventario de Perfeccionismo Infantil (I.P.I.). Psicothema, 24(1), 149-155. Recuperado de http://www.psicothema.com/psicothema.asp?id=3992

Morris, L., \& Lomax, C. (2014). Assessment, development, and treatment of childhood perfectionism: a systematic review. Child and Adolescent Mental Health, 19(4), 225-234. doi: 10.1111/camh.12067

Muñoz, A., López-Mora, C., Portolés, A., y González, J. (2016a). Efectos del perfeccionismo, perseverancia y actividad física sobre factores asociados al rendimiento académico. En J. Gázquez, M. Molero, M. Pérez-Fuentes, M. Simón, A. Barragán y A. Martos (Eds.), Investigación en el ámbito escolar: un acercamiento multidimensional a las variables psicológicas y educativas (Vol. II, pp. 180-185). Almería: ASUNIVEP.

Muñoz, A., López-Mora, C., Portolés, A., y González, J. (2016b). Percepción de estrés e indicadores de perfeccionismo en estudiantes de Secundaria. En J. Castejón (Ed.), Psicología y educación: presente y futuro (pp. 2785-2793). Alicante, España: Asociación Científica de Psicología y Educación (ACIPE). Recuperada de http://rua.ua.es/dspace/handle/10045/64472

O’Connor, R., Rasmussen, S., \& Hawton, K. (2010). Predicting depression, anxiety and self-harm in adolescents: The role of perfectionism and acute life stress. Behaviour Research and Therapy, 48(1), 52-59. doi: 10.1016/j.brat.2009.09.008

Oros, L. (2003). Medición del perfeccionismo infantil: desarrollo y validación de una escala para niños de 8 a 13 ańos de edad. Revista Iberoamericana de Diagnóstico y Evaluación Psicológica, 16(2), 99-112. Recuperado de http://www.aidep. org/03_ridep/R16/R166.pdf

Oros, L. (2004). Incidencia de las prácticas parentales sobre las creencias y los modos de afrontamiento del niño. (Tesis doctoral inédita). Universidad Nacional de San Luis, Argentina.

Oros, L. (2005). Implicaciones del perfeccionismo infantil sobre el bienestar psicológico: orientaciones para el diagnóstico y la práctica clínica. Anales de Psicología, 21(2), 294-303. Recuperado de http://revistas.um.es/analesps/article/ view/26951 
Oros, L., y Vargas-Rubilar, J. (2016). Perfeccionismo infantil: Normalización de una escala argentina para su evaluación. Acción Psicológica, 13(2), 117-126. doi: 10.5944/ap.13.2.17822

Pamies, L., y Quiles, Y. (2014). Perfeccionismo y factores de riesgo para el desarrollo de trastornos alimentarios en adolescentes espańoles de ambos géneros. Anales de Psicología, 30(2), 620-626. doi: 10.6018/analesps.30.2.158441

Parra, A., y Rodrigues, A. (2014). Estrés, perfeccionismo y resiliencia en estudiantes universitarios de Artes. Psicología, 33(2), 81-95. Recuperado de http://saber. ucv.ve/ojs/index.php/rev_ps/article/view/12420

Rice, K., \& Preusser, K. (2002). The Adaptive/Maladaptive Perfectionism Scale (AMPS). Measurement and Evaluation in Counseling and Development, 34(4), 210-222.

Rodríguez-Jiménez., T., Blasco, L., y Piqueras, J. (2014). Relación entre subtipos de sintomatología obsesivo-compulsiva y de perfeccionismo en adolescentes. Psicología Conductual, 22(2), 203-217.

Saboonchi, F., \& Lundh, L. (2003). Perfectionism, anger, somatic health, and positive affect. Personality and Individual Differences, 35(7), 1585-1599. doi: 10.1016/ S0191-8869(02)00382-3

Schweitzer, R., \& Hamilton, T. (2002). Perfectionism and Mental Health in Australian University Students: ¿Is There a Relationship? Journal of College Student Development, 43(5), 684-695. Recuperado de http://eprints.qut.edu.au/3875/

Serppe, M., Chemisquy, S., y Oros, L. (agosto, 2016). Evaluación del perfeccionismo insano en varones y niñas de 9 a 12 años: ¿quiénes están en mayor riesgo de desarrollarlo? Sesión de cartel presentada en el X Congreso Argentino de Salud Mental y III Congreso Regional de la World Federation for Mental Health, Buenos Aires.

Serppe, M., Chemisquy, S., Oros, L., Díaz, J., Barasz, V., Waigel, N., y Ernst, C. (2017). ¿Qué sabemos acerca del perfeccionismo infantil en Argentina? Revista de Psicología, 13(25), 55-69. Recuperado de http://erevistas.uca.edu.ar/index. $\mathrm{php/RPSI/article/view/1066}$

Siegle, D., \& Schuler, P. (2000). Perfectionism differences in gifted middle school students. Roeper Review, 23(1), 39-44. doi: 10.1080/02783190009554060

Stornelli, D., Flett, G., \& Hewitt, P. (2009). Perfectionism, achievement, and affect in children: a comparison of students from gifted, arts, and regular programs. Canadian Journal of School Psychology, 24(4), 267-283. doi: $10.1177 / 0829573509342392$ 
Perfeccionismo en escolares de Caracas: diferencias en función del sexo, tipo de institución educativa y nivel de estrés

Thomson, E. (2017). Perfectionism and self-stigma: The moderating effects of exposure to mental illness. (Tesis de maestría). University of Saskatchewan, Canadá. Recuperada de http://ecommons.usask.ca/handle/10388/7965

Uz-Baş, A. (2011). Dimensions of perfectionism in elementary school-aged children: Association with anxiety, life satisfaction, and academic achievement. Education and Science, 36(162), 261-272. Recuperado de http://egitimvebilim.ted.org.tr/ index.php/EB/article/view/1096

Ventura-León, J., Jara-Avalos, S., Garcia-Pajuelo, C., y Ortiz-Saenz, C. (2018). Validación de una escala de perfeccionismo en niños peruanos. Actualidades en Psicologia, 32(124), 15-32. doi: 10.15517/ap.v32i124.30385

Vicent, M., Aparicio-Flores, M., Inglés, C., Gómez-Nuñez, M., Fernández-Sogorb, A., y Aparisi-Sierra, D. (2017). Perfeccionismo infantil: diferencias en función del sexo y la edad. International Journal of Developmental and Educational Psychology, 3(1), 15-24. doi: 10.17060/ijodaep.2017.n1.v3.970

Yang, H., Hong, C., Tao, X., \& Zhu, L. (2015). Revising the Child and Adolescent Perfectionism Scale A Test of the Four-Factor Structure in a Chinese Sample. Measurement and Evaluation in Counseling and Development, 48(3), 192-203. doi: $10.1177 / 0748175615578733$ 